\title{
Adaptive control of mode conversion by simulated annealing
}

\section{for mode division multiplexing}

\author{
Yi Wang ${ }^{1, ~ a ~}$, Li Gao ${ }^{2, b}$ and Mingying Lan ${ }^{2, c}$ \\ ${ }^{1}$ School of Network Education, Beijing University of Posts and Telecommunications, \\ Beijing 100876, China \\ ${ }^{2}$ School of Digital Media and Design Arts, Beijing University of Posts and Telecommunications, \\ Beijing 100876, China \\ avoid_wy@yeah.net, bgaoli@bupt.edu.cn, 'lanmingying@bupt.edu.cn
}

Keywords: mode conversion, adaptive control, simulated annealing (SA), spatial light modulation (SLM), charge coupled device (CCD)

\begin{abstract}
This paper experimentally implements an adaptive control method that completes conversion between different modes. A charge coupled device (CCD) camera is used to capture the field distribution of the experimental output light. To maximize the correlation between the measured field distribution and the target mode profile, this method uses simulated annealing (SA) algorithm to control the phase modulation (PM) of the experimental input light by a phase-only spatial light modulator (SLM). Experimental results show the conversion is able to achieve between various linearly polarized (LP) modes.
\end{abstract}

\section{Introduction}

In recent years, due to the rapid growth of capacity requirements, spatial division multiplexing (SDM) is becoming a new hot spot in optical communication [1]. SDM uses the spatial dimension to increase the capacity limit of single fiber. And as an implementation of SDM, mode division multiplexing (MDM) makes use of the modes in multimode fiber (MMF) as channels to transmit information. Research shows that it is possible to suppress the crosstalk between modes with future technological developments, thus MDM is great potential [2].

As the basis of MDM, mode conversion is used to achieve data exchange in the future optical network. In the existing study, there are 2 main methods to achieve mode conversion, waveguide structure method [3] and free space optical path method [4]. The method of this paper belongs to the free space optical path method, but it is different from the most methods. The method described here requires no prior knowledge of the optical system and treats the entire optical system as a black box.

The method of this paper is a kind of adaptive control [5] method. The only concerned performance indicator of the optical system is the correlation between the field distribution captured by a charge coupled device (CCD) and the target mode profile. To achieve the desired control target, which is to maximize the correlation, this method produce the corresponding feedback law by simulated annealing (SA) [6] algorithm to control the phase modulation (PM) of the experimental input light by a phase-only spatial light modulator (SLM).

There are five sections in this paper. In Section 2, the experiment setup is introduced. In Section 3, the adaptive control algorithm is described. In Section 4, the experimental results is shown. Finally, there is a conclusion in Section 5.

\section{Experiment setup}

The experiment setup of this paper is shown in Fig. 1.

In the experiment, light is generated from a laser $(1550 \mathrm{~nm})$. Then the light is transmitted to a fiber collimator through a MMF. After being collimated, the light is transmitted to a polarizer through the free space optical path. Next the linearly polarized (LP) light is transmitted to a phase-only SLM (Holoeye, Pluto) through the free space optical path. 
The phase-only SLM approximately has the effective area of $15.36 \mathrm{~mm} \times 8.64 \mathrm{~mm}$ and the number of pixels is $1920 \times 1080$. By estimating the maximum spot size, the effective number of pixels is no more than $400 \times 400$ in the experiment. This area is used to control the PM of the experimental input light.

After being reflected by the phase-only SLM, the light is transmitted to a lens and is captured by the CCD camera in the end through the free space optical path. From the measured intensity profile by the CCD camera, the correlation with the target mode profile is calculated as the performance indicator for adaptive control.

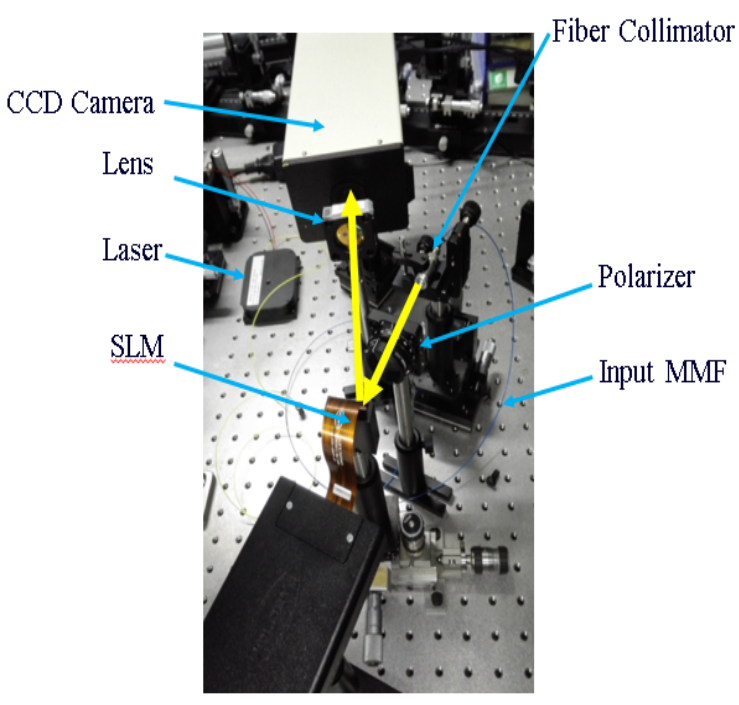

Fig. 1. Experiment setup

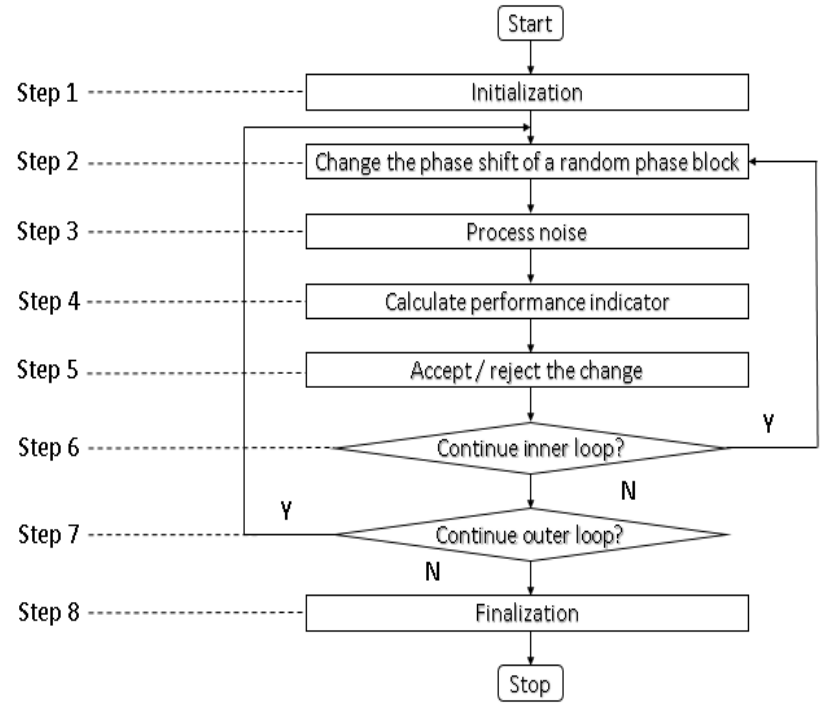

Fig. 2. Adaptive control algorithm flowchart

\section{Adaptive control}

The adaptive control algorithm flowchart of this paper is shown in Fig. 2.

There are 8 steps. Step 1 completes the initialization of the adaptive control algorithm. First, initialize the CCD camera. Initialize the image capture card and capture the first image. Then generate the target mode image matched with the first image. Second, initialize the phase-only SLM. Divide the effective area into $10 \times 10$ phase blocks, which can only produce phase shift of 0 or $\pi$. And initialize all phase blocks with the phase shift of 0 . Third, initialize the parameters of the SA algorithm. $\mathrm{T}_{0}=10^{7}$ means the start temperature and $\mathrm{T}_{\mathrm{f}}=1$ means the stop temperature. $\mathrm{T}_{\mathrm{k}}=\mathrm{T}_{0}$ represents the current temperature and $r=0.95$ is the temperature drop rate of $T_{k} . T_{k}$ and $r$ are used to control the times of the outer loop while $n_{t}=500$ denotes the times of the inner loop.

Step 2 changes the phase shift of a random phase block so that the intensity profile captured by the CCD camera changes simultaneously.

To reduce the impact of the noise on the performance indicator, Step 3 uses median filter (MF) to process the noise. The pixel value $\hat{\mathrm{f}}(\mathrm{x}, \mathrm{y})$ is replaced by the median value of the $3 \times 3$ neighborhood $\mathrm{S}_{\mathrm{xy}}$ :

$$
\hat{\mathrm{f}}(\mathrm{x}, \mathrm{y})=\operatorname{median}_{(\mathrm{s}, \mathrm{t}) \in \mathrm{S}_{\mathrm{xy}}}\{\mathrm{g}(\mathrm{s}, \mathrm{t})\} .
$$

Step 4 calculates the performance indicator, which is the correlation between the intensity profile $\mathrm{I}_{\mathrm{k}}$ captured by the CCD camera and the intensity profile $\mathrm{I}_{0}$ of the target mode: 


$$
f(k)=\frac{\sum\left(\mathrm{I}_{0}(\mathrm{x}, \mathrm{y})-\overline{\mathrm{I}}_{0}\right)\left(\mathrm{I}_{\mathrm{k}}(\mathrm{x}, \mathrm{y})-\overline{\mathrm{I}}_{\mathrm{k}}\right)}{\sqrt{\sum\left(\mathrm{I}_{0}(\mathrm{x}, \mathrm{y})-\overline{\mathrm{I}}_{0}\right)^{2} \sum\left(\mathrm{I}_{\mathrm{k}}(\mathrm{x}, \mathrm{y})-\overline{\mathrm{I}}_{\mathrm{k}}\right)^{2}}} .
$$

where $\overline{\mathrm{I}}_{\mathrm{k}}$ and $\overline{\mathrm{I}}_{0}$ are the average of $\mathrm{I}_{\mathrm{k}}$ and $\mathrm{I}_{0}$.

Step 5 makes the decision whether the change in Step 2 is accepted or rejected by the Metropolis criterion:

$$
p_{i j}=\left\{\begin{array}{cl}
1, & f(j)>f(i) \\
\exp \left(\frac{f(j)-f(i)}{T_{k}}\right), & \text { otherwise }
\end{array} .\right.
$$

Here $p_{i j}$ is the probability that current solution $i$ accepts the new solution $j$ and the current temperature $T_{k}$ is related to $p_{i j}$.

Step 6 determines whether to end the inner loop. If cycle times less than $n_{t}$, cycle times plus 1 and next go to Step 2. Otherwise, set cycle times 0 and next go to Step 7 .

Step 7 determines whether to end the outer loop. If $T_{k}>T_{f}, T_{k}=T_{k} \times r$ and next go to Step 2 . Otherwise, next go to Step 8.

Step 8 completes the finalization of the adaptive control algorithm. Save the results desired and release the resource allocated.

\section{Experimental results}

Before the adaptive control algorithm runs, the initial intensity profile of this paper is shown in Fig. 3 captured by the CCD camera. And after the steps described above, the experimental results are shown in Fig. 4.
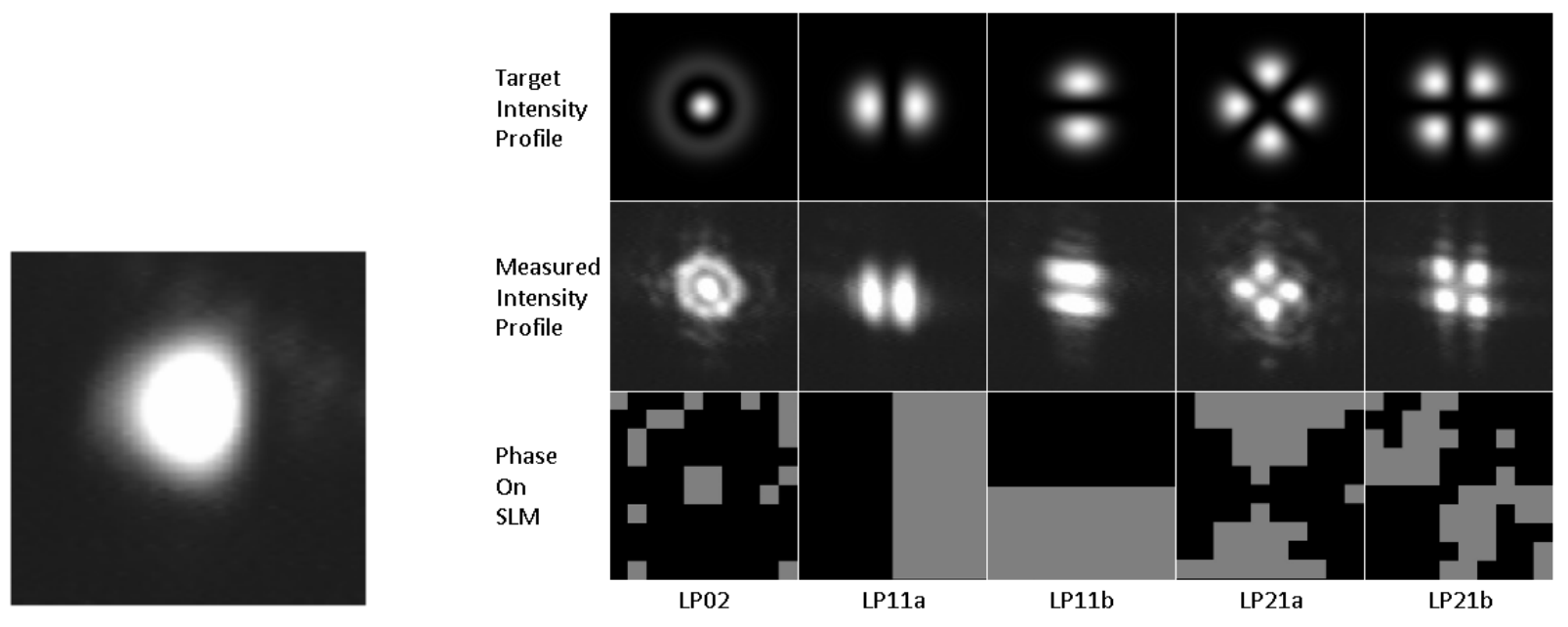

Fig. 3. Initial intensity profile

Fig. 4. Experimental results

The correlation between the intensity profile of the converted mode and the intensity profile of the target mode are 0.681978 (LP02), 0.868725 (LP11a), 0.834541 (LP11b), 0.694744 (LP21a), 0.760707 (LP21b). 


\section{Conclusion}

This paper experimentally demonstrate an adaptive control method to achieve mode conversion. The correlation between the measured field distribution and the target mode profile is used as the feedback and the control law is produced by the SA algorithm to maximize it. The method described here requires no prior knowledge of the optical system. So it can be generalized to the system, which uses CCD camera to capture the filed distribution of the experimental output light and uses SLM to modulation.

\section{Acknowledgements}

This research was supported by the National Basic Research Program of China (973 Program) (2014CB340102), National Natural Science Foundation of China (61271191, 61302085).

\section{References}

[1] Essiambre R. J., Ryf R., Fontaine N. K., et al.: Breakthroughs in Photonics 2012: Space-Division Multiplexing in Multimode and Multicore Fibers for High-Capacity Optical Communication, Ieee Photonics Journal, Vol. 5, No. 2, 2013.

[2] Sillard P., Bigot-Astruc M. and Molin D.: Few-Mode Fibers for Mode-Division-Multiplexed Systems, Lightwave Technology, Journal of, Vol. PP, No. 99(2014) p. 1-1.

[3] Olaya J. C., Ehrlich K., Haynes D. M., et al.: Multimode to single-mode converters: new results on 1-to-61 photonic lanterns, Modern Technologies in Space-and Ground-Based Telescopes and Instrumentation Ii, Vol. 8450(2012).

[4] Carpenter Joel and Wilkinson Timothy D: Precise modal excitation in multimode fibre for control of modal dispersion and mode-group division multiplexing, European Conference and Exposition on Optical Communications, Optical Society of America(2011).

[5] Yu, W., DeLellis, P., Chen, G., di Bernardo, M., \& Kurths, J.: Distributed adaptive control of synchronization in complex networks.Automatic Control, IEEE Transactions on, Vol. 57, No. 8 (2012), p. 2153-2158.

[6] Carpenter Joel and Wilkinson Timothy D.: Graphics processing unit-accelerated holography by simulated annealing, Optical Engineering, Vol. 49, No. 9(2010)p. 095801-095807. 